\title{
Passive Wake Vortex Control
}

\author{
J. M. Ortega
}

October 18, 2001

U.S. Department of Energy

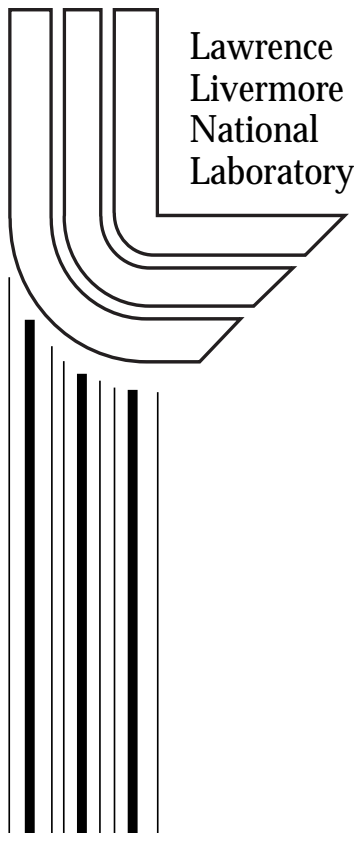




\section{DISCLAIMER}

This document was prepared as an account of work sponsored by an agency of the United States Government. Neither the United States Government nor the University of California nor any of their employees, makes any warranty, express or implied, or assumes any legal liability or responsibility for the accuracy, completeness, or usefulness of any information, apparatus, product, or process disclosed, or represents that its use would not infringe privately owned rights. Reference herein to any specific commercial product, process, or service by trade name, trademark, manufacturer, or otherwise, does not necessarily constitute or imply its endorsement, recommendation, or favoring by the United States Government or the University of California. The views and opinions of authors expressed herein do not necessarily state or reflect those of the United States Government or the University of California, and shall not be used for advertising or product endorsement purposes.

This is a preprint of a paper intended for publication in a journal or proceedings. Since changes may be made before publication, this preprint is made available with the understanding that it will not be cited or reproduced without the permission of the author.

This work was performed under the auspices of the United States Department of Energy by the University of California, Lawrence Livermore National Laboratory under contract No. W-7405-Eng-48.

This report has been reproduced directly from the best available copy.

Available electronically at http://www.doc.gov/bridge

Available for a processing fee to U.S. Department of Energy

And its contractors in paper from

U.S. Department of Energy

Office of Scientific and Technical Information

P.O. Box 62

Oak Ridge, TN 37831-0062

Telephone: (865) 576-8401

Facsimile: (865) 576-5728

E-mail: reports@adonis.osti.gov

Available for the sale to the public from

U.S. Department of Commerce

National Technical Information Service

5285 Port Royal Road

Springfield, VA 22161

Telephone: (800) 553-6847

Facsimile: (703) 605-6900

E-mail: orders@ntis.fedworld.gov

Online ordering: http://www.ntis.gov/ordering.htm

OR

Lawrence Livermore National Laboratory

Technical Information Department's Digital Library

http://www.llnl.gov/tid/Library.html 


\author{
Passive Wake Vortex Control \\ by \\ Jason M. Ortega \\ University of California \\ Lawrence Livermore National Laboratory \\ P.O. Box 808 L-206 \\ Livermore, CA 94551 \\ ortega17@llnl.gov
}

\title{
1 Motivation
}

The collapse of the Soviet Union and ending of the Cold War brought about many significant changes in military submarine operations. The enemies that the United States Navy faces today and in the future will not likely be superpowers armed with nuclear submarines, but rather smaller, rogue nations employing cheaper diesel/electric submarines with advanced air-independent propulsion systems. Unlike Cold War submarine operations, which occurred in deep-water environments, future submarine conflicts are anticipated to occur in shallow, littoral regions that are complex and noisy. Consequently, non-acoustic signatures will become increasingly important and the submarine stealth technology designed for deep-water operations may not be effective in these environments. One such non-acoustic signature is the surface detection of a submarine's trailing vortex wake. If a submarine runs in a slightly buoyant condition, its diving planes must be inclined at a negative angle of attack to generate sufficient downforce, which keeps the submarine from rising to the surface. As a result, the diving planes produce a pair of counter-rotating trailing vortices that propagate to the water surface (Figure 1a). In previous deep-water operations, this was not an issue since the submarines could dive deep enough so that the vortex pair became incoherent before it reached the water surface. However, in shallow, littoral environments, submarines do not have the option of diving deep and, hence, the vortex pair can rise to the surface and leave a distinct signature $[5,6,9,18,19,20,22]$ that might be detectable by synthetic aperture radar. Such detection would jeopardize not only the mission of the submarine, but also the lives of military personnel on board.

There has been another attempt to solve this problem and reduce the intensity of trailing vortices in the wakes of military submarines. The research of Quackenbush et al. [14, 15, 16,17 over the past few years has been directed towards an idea called "vortex leveraging." This active concept works by placing shape memory alloy (SMA) control surfaces on the submarine's diving planes and periodically oscillating them. The modulated control vortices generated by these surfaces interact with the tip vortices on the diving planes, causing an 
instability to rapidly occur. Though several numerical simulations have been presented, experimental verification does not appear to be available in the open literature.

We address this problem through a concept called passive wake vortex control (PWVC), which has been demonstrated to rapidly break apart a trailing vortex wake and render it incoherent. PWVC functions by introducing unequal strength, counter-rotating control vortices next to the tip vortices (Figure 1b). The presence of these control vortices destabilizes the vortex wake and produces a rapidly growing wake instability.

\section{Experimental Setup}

PWVC has been investigated through a series of experiments at the U.C. Berkeley Richmond Field Station towing tank facility [10, 11, 12, 13]. A modular wing is used to generate the trailing vortices. Depending on the type of lift distribution desired, different tabs and flaps are attached to the trailing edge of the wing. The modular wing has a span, $b$, of $40 \mathrm{~cm}$ and nominal chord, $c$, of $6.67 \mathrm{~cm}$. The wing is constructed of $1 / 8$ " thick stainless steel sheet metal that has been rolled to give it a camber radius of $17 \mathrm{~cm}$. Two different types of wings are used in these experiments to provide a comparison of vortex wakes with and without PWVC. For the baseline case of a wing without PWVC, a rectangular planform wing is used (Figure 2a). For the wing with PWVC, outboard, triangular-shaped flaps are utilized to generate the control vortices (Figure $2 \mathrm{~b}$ ). Each of these flaps has a span of $d=b / 4$ and an extensional size of $0.5 c$.

\section{Flow Visualization}

Volumetric flow visualization of the trailing vortex wakes is achieved by injecting florescent dye into the control and tip vortices (Figure 3). The dye motions are then recorded with several cameras located in and around the 200' long towing tank. The flow visualization measurements for the baseline, rectangular wing are shown in Figure $4 \mathrm{a}$, where $x / b$ in these figures is the downstream distance behind the wing in terms of wingspans. For this run, the wing velocity is $U_{o}=500 \mathrm{~cm} / \mathrm{s}$ and the angle of attack of the wing is $\alpha=3^{\circ}$. The carriage and wing are visible at $x / b=0$. Over the course of these measurements, the vortex wake without PWVC behaves in a very stable, two-dimensional fashion. It is not until the vortex pair approaches the bottom of the tank that it begins to show signs of a long-wavelength, Crow-like instability [4]. On the other hand, the vortex wake with PWVC behaves in a completely different manner. Figure $4 \mathrm{~b}$ shows a sequence of images in the wake of the triangular-flapped wing at various downstream locations, where $U_{o}=500 \mathrm{~cm} / \mathrm{s}$ and 
$\alpha=2^{\circ}$. For this experimental run, the counter-rotating control vortices are $37 \%$ as strong as the tip vortices. After their initial formation, the control and tip vortices orbit outwardly away from the wing centerline. At approximately 15 spans or $135^{\circ}$ through the orbit period, stationary, sinuous instability waves are observed to rapidly develop on the control vortices. The wavelength of the instability is on the order of one wingspan or four times the separation distance between the flap and tip vortices. The wave amplitudes quickly grow such that the control and tip vortices on the port-side make contact at about 20 spans and those on the starboard-side at 25 spans. As the instability progresses, the control vortices wrap around the tip vortices, forming " $\Omega$ "-shaped hoops. The spiral "feet" of these hoops behave like vortex rings and advect themselves inward toward each other. This extends the hoops in the vertical direction. By 45 spans, the hoops are oriented vertically and are flung across the center of the wake. As the hoops approach the wake centerline, they pinch off into vortex rings, occasionally colliding with rings from the opposite side of the airfoil. The vortex rings that make it to the opposite of the wake collide with the remnants of the tip vortices. Figure 5 shows flow visualization images from the same experimental run, but from the vantage point of a submerged camera. The measured instability wavelengths along the control vortices are shown to agree favorably with those predicted by two- and four-vortex linear instability analyses (Appendix A), demonstrating that these analytical models are capturing the essential physics of the instability growth.

\section{PIV Measurements}

Although the flow visualization provides a good qualitative description of the instability between the control and tip vortices, it does not allow for the measurement of other quantities, such as the circulation strengths, kinetic energy of the vortex wake, or the vortex structure. Therefore, two-dimensional particle imaging velocimetry (PIV) measurements are made in the vortex wakes with and without PWVC. For the wakes without PWVC, both the starboard- and port-side halves of the wake are measured. However, since the wakes with PWVC become so spread out due to the instability, only the starboard-half of the wake is imaged for the PIV measurements. Figure 6a shows the trajectories for the starboard- and port-side tip vortices for the baseline rectangular wing without PWVC. For this particular run, the towing speed of the wing is $U_{o}=500 \mathrm{~cm} / \mathrm{s}, \alpha=3^{\circ}$, and $R e_{c}=334,000$. Consistent with the flow visualization observations, the wake without PWVC descends quietly throughout the entire time of measurement and does not exhibit any signs of an instability or vortex breakup. In contrast, the unsteady, three-dimensional, and dynamic nature of the wakes with PWVC is strongly evident in the PIV data. Figures 6b-c demonstrate the 
vorticity centroid trajectories for two of the experimental runs with PWVC. In Figure 6b, $U_{o}=500 \mathrm{~cm} / \mathrm{s}, \alpha=2^{o}, R e_{c}=334,000$, and $\Gamma_{c} / \Gamma_{t}=-0.37$ and in Figure $6 \mathrm{c}, U_{o}=500 \mathrm{~cm} / \mathrm{s}$, $\alpha=-1^{o}, R e_{c}=334,000$, and $\Gamma_{c} / \Gamma_{t}=-0.53$, where $\Gamma_{c}$ and $\Gamma_{t}$ are the circulation strengths of the control and tip vortices, respectively. The curved black lines are the trajectories of the control and tip vortices prior to the non-linear vortex interactions that occur around $x / b=38$. The thick, black line is the position of the overall vorticity centroid for $x / b \leq$ 38 , while the thick gray line is the position of the overall vorticity centroid for $x / b>38$. The measurements in Figure $6 \mathrm{~b}$ are made up to $x / b=240$ and those in Figure $6 \mathrm{c} x / b=$ 330. For both of these runs, the overall vorticity centroids behave in a manner quite unlike that of the wake without PWVC. In particular, the overall vorticity centroid for the run with $\Gamma_{c} / \Gamma_{t}=-0.53$ lingers in the vicinity of the tip vortex's final location. This is not to say that the vortex wake has stopped descending completely, but, rather, that it's vertical descent velocity has been reduced due to the lateral spreading of momentum caused by the non-linear vortex interactions. A review of particle-streak data for this run revealed that there is a small, but finite, amount of downwash in the vortex wake at $x / b=330$.

One means of assessing the three-dimensionality of vortex wakes with PWVC is to look at the two-dimensional kinetic energy from the PIV measurements. Figure 7 illustrates the trends in the two-dimensional kinetic energy for a vortex wake without PWVC and two vortex wakes with PWVC. The variable $K$.E.tot.avg. in Figure 7 is the average total kinetic energy over each of the runs. For the wake without PWVC (Figure 7a), the two-dimensional kinetic energy varies only gradually over the entire run, indicating that the vortex wake is behaving in a two-dimensional fashion. However, the two-dimensional kinetic energy in the vortex wakes with PWVC (Figure 7b-c) rapidly drops as soon as the instability tilts and disrupts the vortices in the PIV measurement plane. For larger values of $\left|\Gamma_{c} / \Gamma_{t}\right|$ (Figure $7 \mathrm{c}$ ), the two-dimensional kinetic energy decays more quickly and remains at a value approximately one-fifth of that at $x / b=0$ for the remainder of the run.

\section{Computer Simulations}

In addition to the experimental and theoretical (Appendix $\mathrm{A}$ ) analyses, the vortex wakes with PWVC are investigated with computer simulations $[1,2]$. The results of these simulations provide three-dimensional velocity and vorticity fields, which could not be measured with the flow visualization or PIV techniques. The simulations of the vortex wakes are done with a spectral Navier-Stokes solver in cylindrical coordinates $[7,8]$. Since the code is periodic along the length of the vortices, it is ideally suited for modeling the trailing vortices in the far wakes of the wings. Figure 8 shows surfaces of constant vorticity for a simulation of 
a wake similar to those in Figures $4 \mathrm{~b}, 5,6 \mathrm{~b}$, and $7 \mathrm{~b}$, where $\Gamma_{c} / \Gamma_{t}=-0.37$. To initialize the

flow, the linear stability analysis for a four-vortex wake (Appendix A) is used to determine the most unstable eigenmode. At $t=0$, the control and tip vortices are perturbed with this eigenmode. Since this one eigenmode is singled out and perturbed, it must be kept in mind that this simulation is not a true measure of the effectiveness of PWVC. A better initial condition would be to perturb the control and tip vortices with small amplitude noise at $t=$ 0 and let the flow evolve from there. However, these results are merely shown to demonstrate the capability of this code and to provide an approximate comparison with the experimental measurements. It can be seen in Figure 8 that the control vortices quickly develop sinuous waves along their lengths. As is also seen in the flow visualization data, the control vortices wrap around the tip vortices, leading to the formation of vortex hoops that are flung across the center of the wake.

\section{Application of PWVC to Navy Submarines}

Due to its ability to rapidly disrupt the coherence of trailing vortices, PWVC may be an effective mechanism for reducing the surface signature of a submarine's vortex wake. Figures 9 and 10 demonstrate how PWVC might be applied to the diving planes on a submarine. The outboard flaps on the diving planes would be retractable, allowing the submarine commander to deploy or retract them as needed.

PWVC offers several unique advantages as a wake alleviation concept for submarines. The first is that it is completely passive. Requiring no oscillating flaps or other active control mechanisms, PWVC functions by simply placing oppositely-signed control vortices near the tip vortices and using their energy to disrupt the coherence of the wake. If PWVC were employed in submarines, there would be fewer concerns of maintaining it against structural fatigue than there would be for an active system. The second advantage of PWVC is that, although it is passive, its design does allow for the non-linear control of the vortex wake evolution. By varying the relative strength of the control vortices, it has been shown that the behavior of the vortex wake can be widely altered. For weaker control vortices, there is a large exchange of vortex hoops across the airfoil centerline. For stronger control vortices, the break up of the trailing vortices is confined to each side of the wake and the sinuous instability leads to an upwards ejection of vortex rings. Therefore, depending on the type of behavior desired in the vortex wake, the strength of the control vortices can be adjusted accordingly. 


\section{Future Research Directions}

Although significant progress has been made in demonstrating PWVC and understanding the physical mechanisms that cause the wake vortex instability, there are still several unanswered questions that need to be addressed before PWVC can be applied to military submarines.

1. How much can PWVC reduce the surface signature of a vortex wake? During the course of study on PWVC, these types of measurements were beyond the scope of the current research project. Consequently, it is necessary to make measurements and calculations to compare the free-surface interactions of vortex wakes with and without PWVC.

2. What values of $\Gamma_{c} / \Gamma_{t}$ and $d$ minimize the free-surface signature of a vortex wake with PWVC?

3. What are the drag and acoustic penalties for deploying outboard flaps on the diving planes?

4. Are these penalties acceptable for the successful performance of a submarine?

5. Do the wakes from the submarine mast, hull, and propeller reduce the effectiveness of PWVC?

6. Are there any important Reynolds number effects that might limit the effectiveness of PWVC? Since these measurements were done at Reynolds numbers orders of magnitude less than those of an actual submarine, it is necessary to determine if there are any significant changes in PWVC as the Reynolds number is increased.

Once each of these questions and, perhaps, others are answered, it will then be possible to determine whether or not PWVC is a technology that can significantly enhance submarine operations in littoral environments.

\section{A Appendix: Linear Stability Theory}

To understand the physical mechanisms that cause the instability in PWVC, a simplified stability analysis, similar to that of Widnall [21], is done for a single, counter-rotating control/tip vortex pair. The vortices are represented by infinitely long vortex filaments, the cross sections of which are shown in Figure 11a. If one of the vortices (vortex 2) is sinusoidally displaced in the $x$-direction with a long wavelength perturbation of wavenumber $k$, the question arises as to whether or not the perturbation amplitude, $r$, will grow or decay in 
time. To answer this question, it is necessary to determine how the rate of strain field about vortex 2 interacts with the self-induced rotation rate of vortex 2 . The rate of strain field is simply the motion of the fluid relative to a coordinate system that is fixed with respect to vortex 2 (Figure 12a). The self-induced rotation rate of vortex 2 is the angular velocity, $\varpi(k)$, of the sinusoidal perturbation on vortex 2 . When the long wavelength, sinusoidal perturbation is placed on vortex 2 , the perturbation does not remain stationary, but rather spins about the vortex axis in a direction opposite (retrograde) to that of the flow about vortex 2 (Figure 12b). An instability occurs when the azimuthal component, $u_{\theta}$, of the rate of strain field is equal and opposite to the vortex self-induced angular velocity, $\varpi(k) r$. This results in the perturbation on vortex 2 becoming fixed at one orientation, $\theta_{o}$ (Figure 12a). There, the radial component, $u_{r}$, of the rate of strain field causes the perturbation amplitude to radially diverge in an exponential fashion. Consequently, the vortex pair is unstable to perturbations of this wavenumber, $k$.

Having described the physical means by which an instability can occur, a more complete linear stability analysis is performed on a single vortex pair by including perturbations that may arise on the other vortex (vortex 1). Crow [4] conducted a similar type of analysis for equal strength, counter-rotating vortex pairs $\left(\Gamma_{1}=-\Gamma_{2}\right)$. Bristol [1] later extended Crow's work to include arbitrary strength vortices $\left(\Gamma_{1} \neq \Gamma_{2}\right)$. The primary difference in Bristol's calculation is that it accounts for the fact that the arbitrary strength vortices orbit about one another at an angular velocity of $\Omega=\left(\Gamma_{1}+\Gamma_{2}\right) /\left(2 \pi d^{2}\right)$. Following the method of Bristol, instability growth rate curves are computed for arbitrary strength, counter-rotating vortex pairs. The results of these calculations demonstrate that counter-rotating vortex pairs with disparate strength vortices have maximum growth rates that are larger than that of the Crow instability $\left(\Gamma_{1}=-\Gamma_{2}\right)$. Furthermore, the wavelength corresponding to the most unstable mode for unequal strength, counter-rotating vortex pairs is shorter than that of the Crow instability .

Since the wake of the triangular-flapped wing is comprised of two counter-rotating vortex pairs, an additional linear stability analysis is performed to account for the presence of the other vortex pair. Crouch [3] conducted a similar type of analysis for co-rotating vortex pairs. Consequently, his linear stability theory is implemented, but is broadened to include counter-rotating control and tip vortices. A schematic of the four-vortex system is shown in Figure 13a. Comparing the growth rate curves (Figure 13b) of the four-vortex system with that of a single vortex pair (vortices 2 and 4 ) shows that they are very similar. This indicates that the most unstable mode of the four-vortex system is driven by the instability of the individual unequal strength, counter-rotating vortex pairs. Another observation that can be 
made is that the most unstable mode for the four-vortex system has a growth rate that is substantially larger and occurs at a wavenumber that is higher than those of the Crow-like instability. Therefore, as observed experimentally, these instabilities arise much sooner than the Crow-like instability and have a wavelength that is distinctly shorter.

This work was performed under the auspices of the U.S. Department of Energy by the University of California, Lawrence Livermore National Laboratory under Contract No. W7405-Eng-48.

\section{References}

[1] Bristol, R. L. 2000 Co-operative wake vortex instabilities. Ph.D. Dissertation, University of California, Berkeley.

[2] Bristol, R. L., Ortega, J. M. \& Savaş, Ö. 2001 Co-operative wake vortex instabilities. In preparation for submittal to the J. Fluid Mech.

[3] Crouch, J. D. 1997 Instability and Transient Growth for Two Trailing-Vortex Pairs, Journal of Fluid Mechanics, 350, 311-330.

[4] Crow, S. C. 1970 Stability theory for a pair of trailing vortices. AIAA Journal, 8, No. $12,2172-2179$.

[5] Dequan, Y. \& Tryggvason, G. 1990 The free-surface signature of unsteady, twodimensional vortex flows. Journal of Fluid Mechanics, 218, 547-572.

[6] Marcus, D.L. \& Berger S.A. 1989 The interaction between a counter-rotating vortex pair in vertical ascent and a free surface. Physics of Fluids A, 1, No. 12, 1988-2000.

[7] Matsushima, T. 1995 Spectral methods in polar coordinates with an application to the stability of a trailing vortex. Ph.D. Dissertation, University of California, Berkeley.

[8] Matsushima, T. \& Marcus, P.S. 1997 A spectral method for unbounded domains. Journal of Computational Physics, 137, 321-345.

[9] Ohring, S. \& Lugt, H.J. 1991 Interaction of a viscous vortex pair with a free surface. Journal of Fluid Mechanics, 227, 47-70.

[10] Ortega, J. M. \& SAvaş, Ö. 2001 A rapidly growing instability mode in trailing multiple-vortex wakes. AIAA J., 39, 750-754.

[11] OrtegA, J. M. 2001 Stability characteristics of counter-rotating vortex pairs in the wakes of triangular-flapped airfoils. Ph.D. Dissertation, University of California, Berkeley. 
[12] Ortega, J. M., Bristol, R. L. \& SAvaŞ, Ö. 2001 Instability of unequal strength counter-rotating vortex pairs. In preparation for submittal to the J. Fluid Mech.

[13] Ortega, J. M., Bristol, R. L. \& SAvaş, Ö. 2001 Wake alleviation properties of triangular-flapped wings. In preparation for submittal to the AIAA J.

[14] Quackenbush, T.R., Bilanin, A.J., McKillip, R.M., Jr. 1996 Vortex Wake Control Via Smart Structures Technology, (Smart Structures and Materials 1996, Industrial and Commercial Applications of Smart Structure Technologies, 27-29, February 1996) Proceedings of the SPIE - The International Society for Optical Engineering, 2721, 78-92.

[15] Quackenbush, T.R., Bilanin, A.J., Batcho, P.F., McKillip, R.M., Jr., Carpenter, B.F. 1997 Implementation of Vortex Wake Control Using SMA-Actuated Devices, (Smart Structures and Materials 1997, Industrial and Commercial Applications of Smart Structure Technologies, 4-6, March 1997) Proceedings of the SPIE - The International Society for Optical Engineering, 3044, 134-146.

[16] Quackenbush, T.R., Batcho, P.F., Bilanin, A.J., Carpenter, B.F. 1998 Design, Fabrication, and Test Planning for an SMA-Actuated Vortex Wake Control System," (Smart Structures and Materials 1998, Industrial and Commercial Applications of Smart Structure Technologies, 3-5, March 1998) Proceedings of the SPIE - The International Society for Optical Engineering, 3326, 259-271.

[17] Quackenbush, T.R., Bilanin, A.J., Carpenter, B.F. 1999 Test Results for an SMAActuated Vortex Wake Control System," (Smart Structures and Materials 1999, Industrial and Commercial Applications of Smart Structure Technologies, March 1999) Proceedings of the SPIE - The International Society for Optical Engineering, 3674, 84-94.

[18] Sarpkaya, T. \& Suthon, P. 1991 Interaction of a vortex couple with a free surface. Experiments in Fluids, 11, 205-217.

[19] Stefanick, T. 1988 The nonacoustic detection of submarines. Scientific American, 258, No. 3, 41-47.

[20] Telste, J.G. 1989 Potential flow about two counter-rotating vortices approaching a free surface. Journal of Fluid Mechanics, 201, 259-278.

[21] Widnall, S. E., Bliss, D. B., Tsai, C.-Y. 1974 The Instability of Short Waves on a Vortex Ring, Journal of Fluid Mechanics, 66, No. 1, 35-47.

[22] Willmarth, W.W., Tryggvason, G., Hirsa, A., \& Yu, D. 1989 Vortex pair generation and interaction with a free surface. Physics of Fluids A, 1, No. 2, 170-172. 
(a)

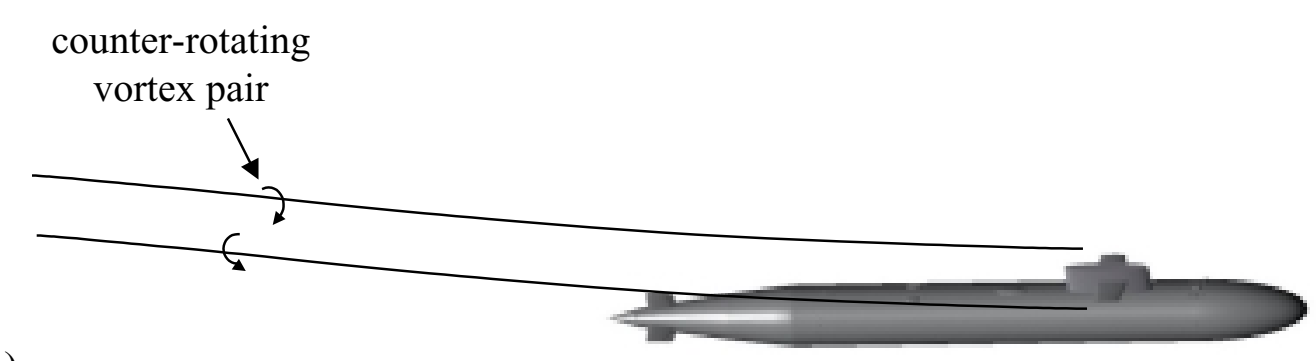

(b)

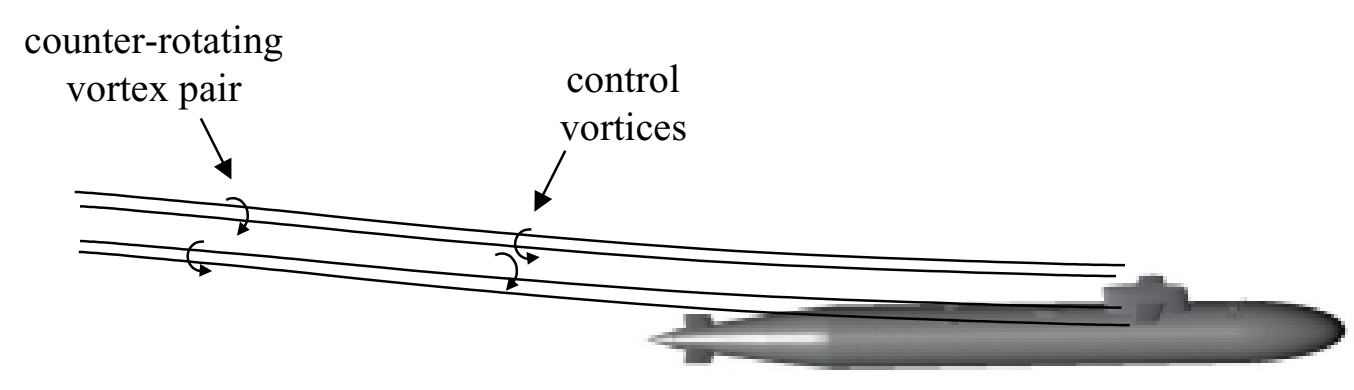

Figure 1: (a) Counter-rotating vortices in the wake of a submarine. (b) A vortex wake with PWVC in which control vortices are produced next to each of the diving plane tip vortices. The control vortices have a sense of rotation that is opposite of that of the nearby tip vortices.

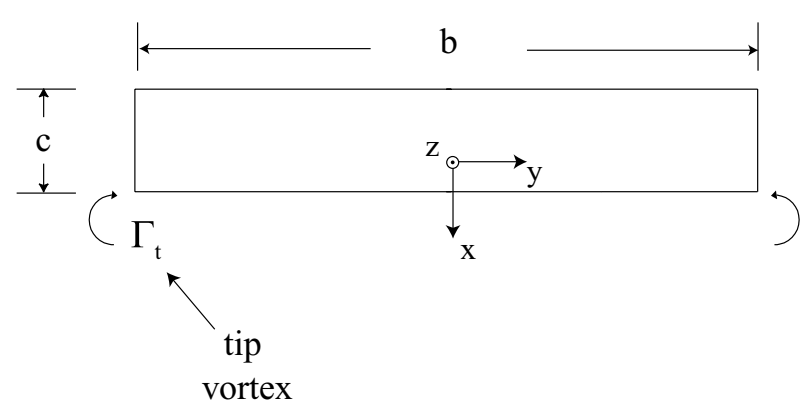

(a)

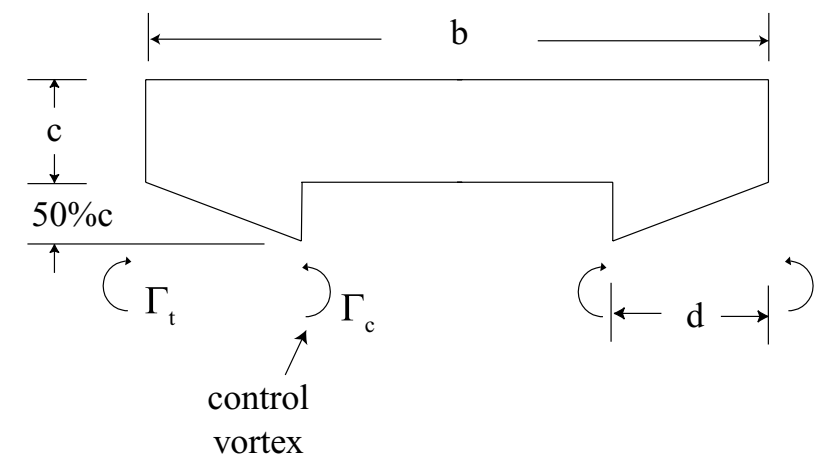

(b)

Figure 2: (a) Rectangular wing used in the towing tank experiments. The wing has a span of $b=40 \mathrm{~cm}$ and chord of $c=6.67 \mathrm{~cm}$ and produces a vortex wake comprised of two counter-rotating tip vortices of strength $\Gamma_{t}$. (b) Triangular-flapped wing used for PWVC. The triangular flaps have span of $d=b / 4$ and an extensional width of $0.5 c$. The control vortices generated by the triangular flaps rotate in the opposite direction as the nearby tip vortices and have strengths of $\Gamma_{c}$. 


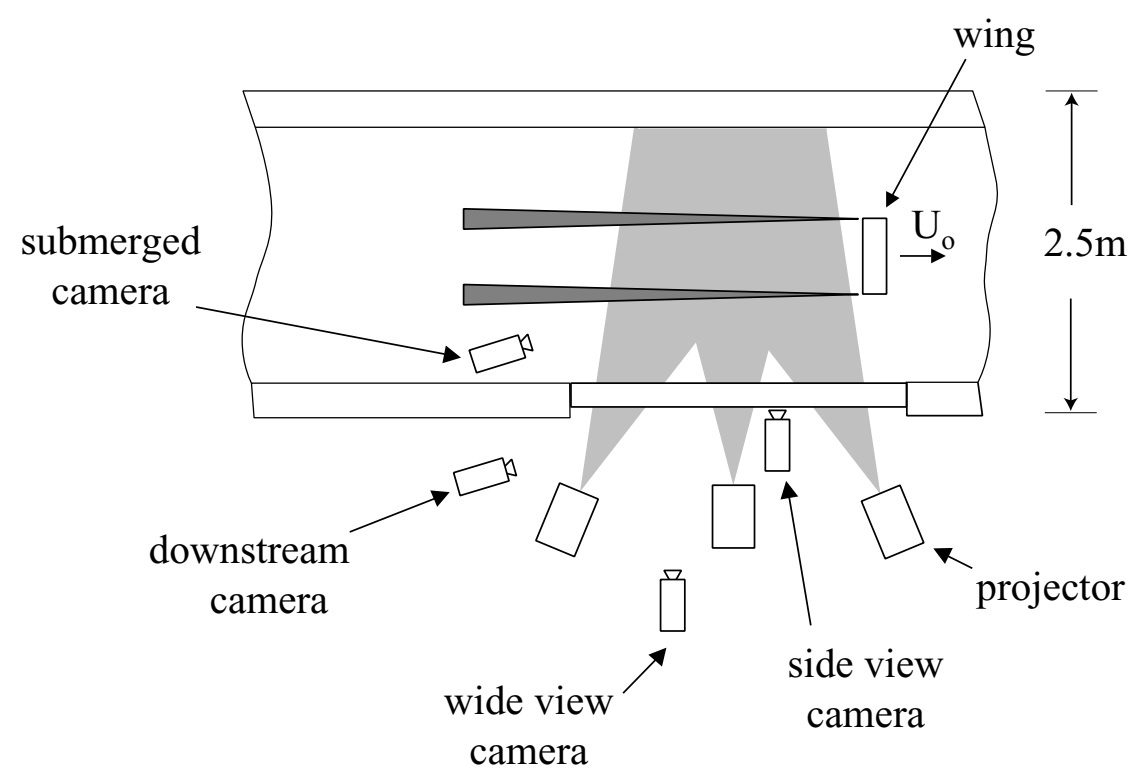

Figure 3: Setup for the flow visualization measurements done in the towing tank facility. As the rectangular or triangular flapped wings are towed down the length of the tank, florescent dye is released into the tip and control vortices. Projectors are used to illuminate the flow field while several cameras record the dye motions. 

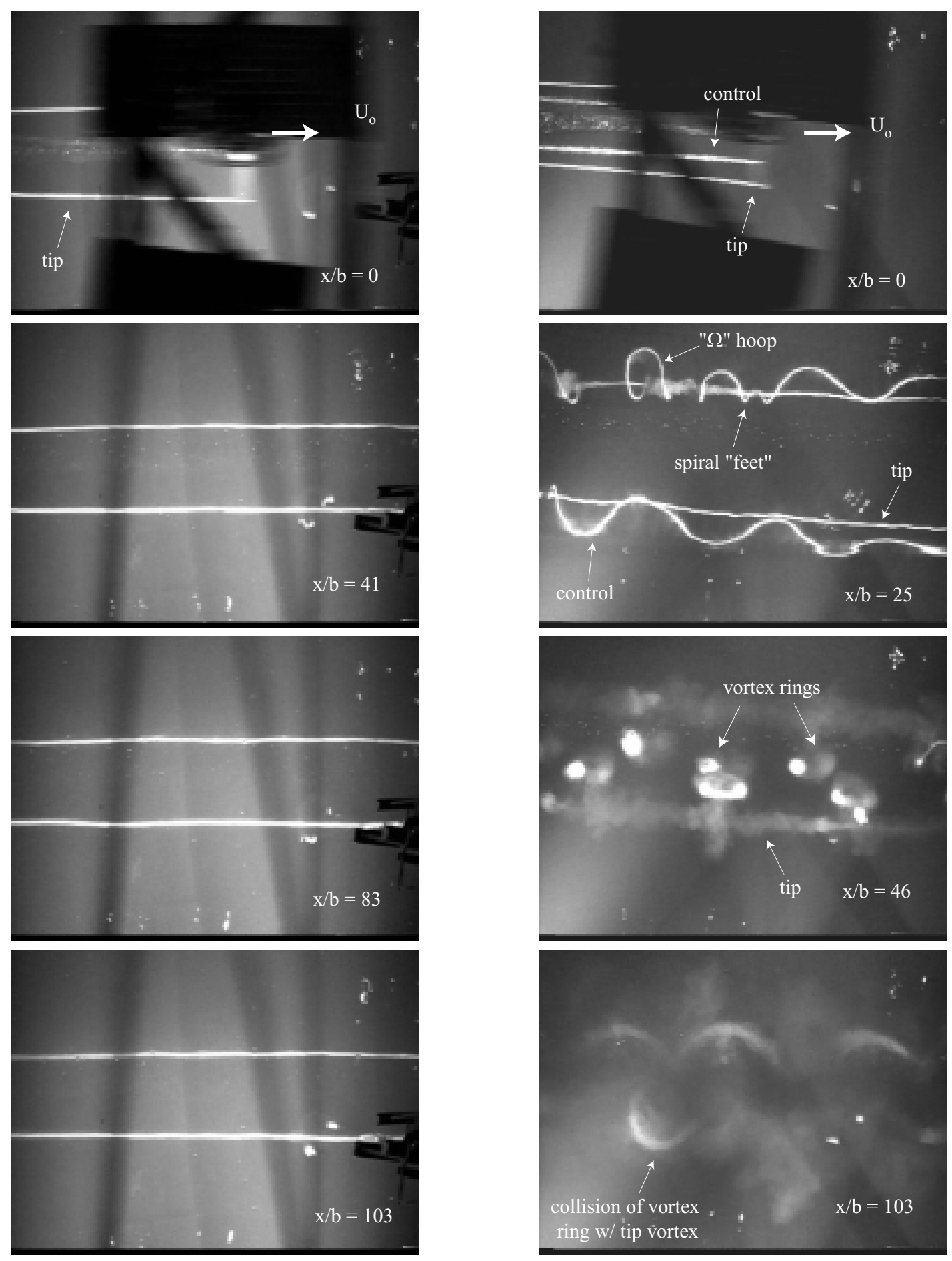

(a)

(b)

Figure 4: (a) Top view of the trailing vortex wake of the baseline rectangular wing. At $x / b=0$, the carriage and wing are visible. For this view, the camera is located about 6' above the surface of the water. (b) Trailing vortex wake with PWVC in which the unequal strength, counter-rotating control vortices are $37 \%$ as strong as the tip vortices. 

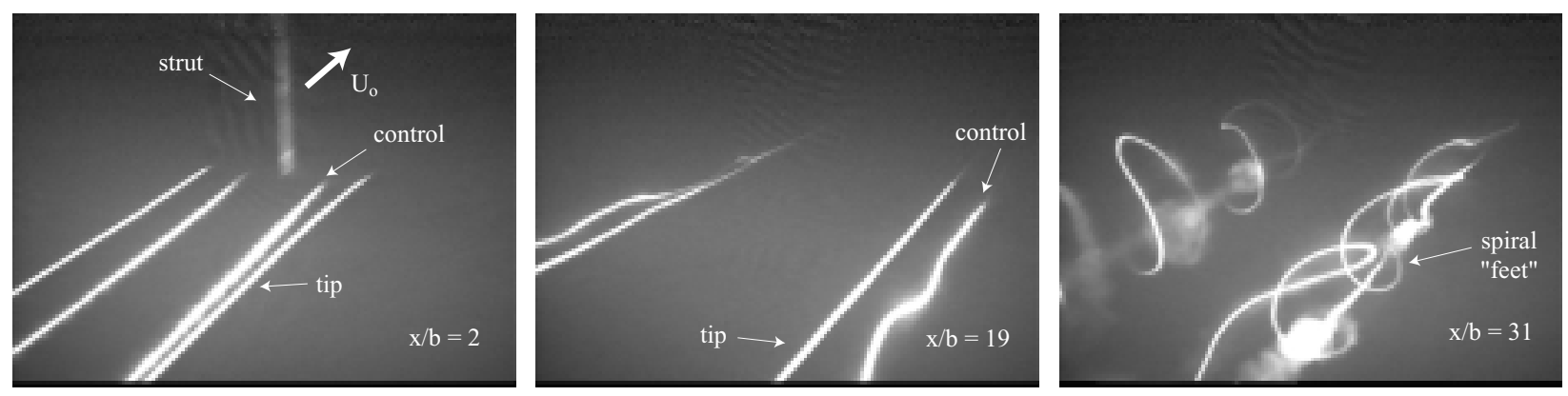

Figure 5: Flow visualization images from a downstream view of the vortex wake shown in Figure 4b.

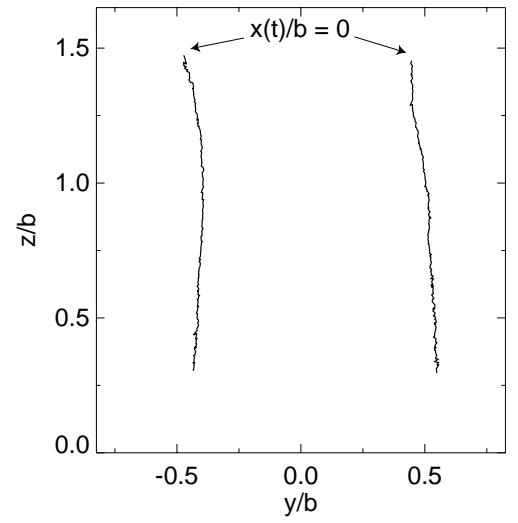

(a)

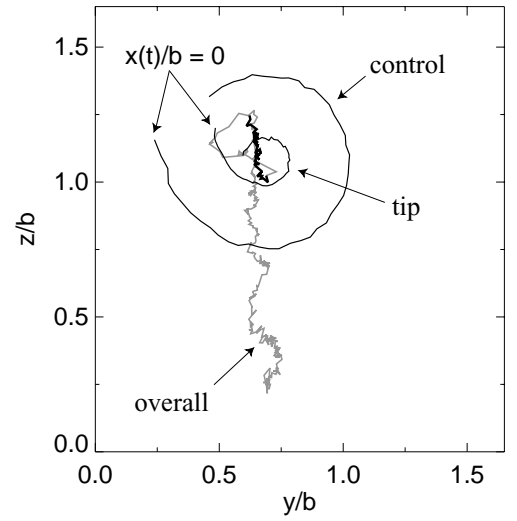

(b)

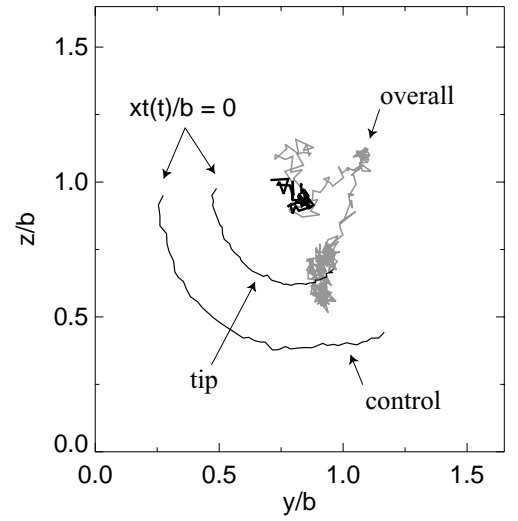

(c)

Figure 6: (a) Trajectories of the vorticity centroids in the wake of the baseline rectangular wing. The label $x(t) / b=0$ indicates the position of the vortices just behind the wing. (b) Trajectories of the starboard tip and control vortices and the overall vorticity centroid for a wake with PWVC in which $\Gamma_{c} / \Gamma_{t}=-0.37$. (c) Trajectories of the starboard tip and control vortices and the overall vorticity centroid for a wake with PWVC in which $\Gamma_{c} / \Gamma_{t}=-0.53$. Prior to the first signs of the wake instability, the paths of the tip and control vortices are indicated by the curved black lines and the overall vorticity centroid by the thick black lines. The thick gray lines are the paths of the overall vorticity centroids after instability becomes evident. 


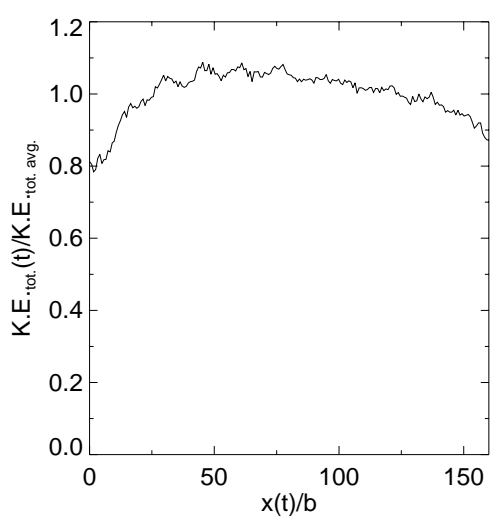

(a)

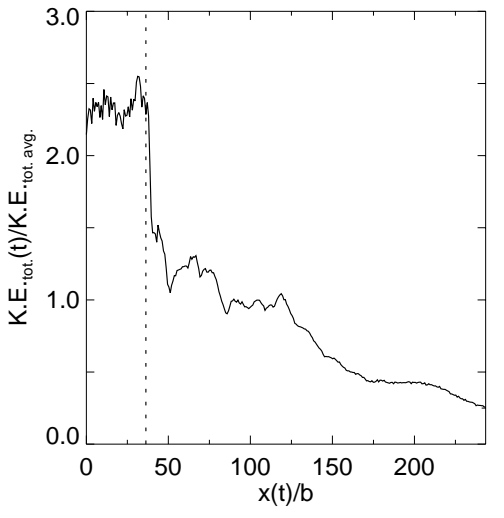

(b)

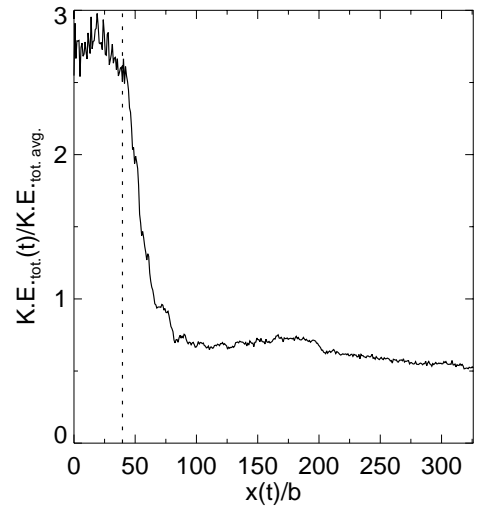

(c)

Figure 7: Two-dimensional kinetic energy of vortex wakes with and without PWVC. (a) The rectangular wing is towed at $U_{o}=500 \mathrm{~cm} / \mathrm{s}$ and $\alpha=3^{\circ}$. (b) The triangular-flapped wing is towed at $U_{o}=500 \mathrm{~cm} / \mathrm{s}$ and $\alpha=2^{o}$ resulting in $\Gamma_{c} / \Gamma_{t}=-0.37$. (c) The triangular-flapped wing is towed at $U_{o}=500 \mathrm{~cm} / \mathrm{s}$ and $\alpha=-1^{o}$ resulting $\Gamma_{c} / \Gamma_{t}=-0.56$. The variable $K$.E.tot.avg. is the average total kinetic energy over each of the runs.

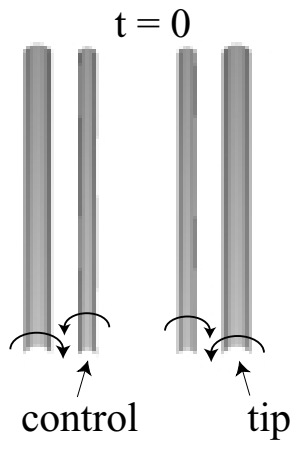

$\mathrm{t}=126$

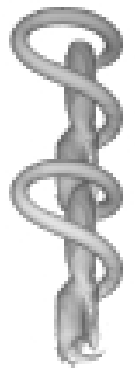

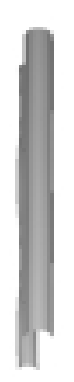

$\mathrm{t}=52$

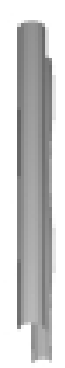

$\mathrm{t}=150$
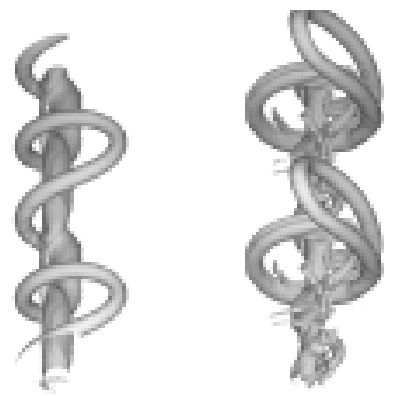

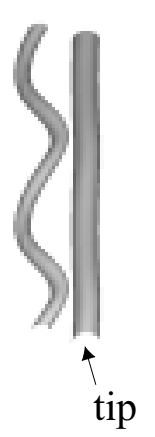

$\mathrm{t}=100$

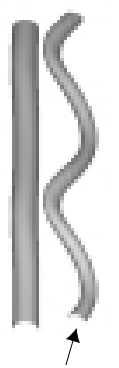

control
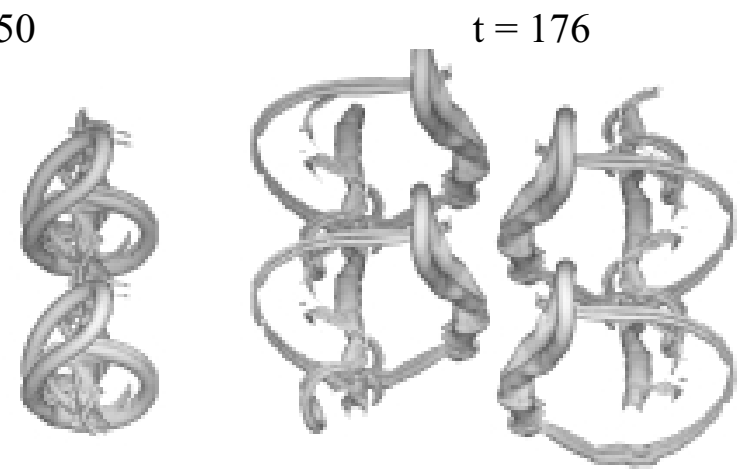

Figure 8: Surfaces of constant vorticity for a wake with PWVC using a spectral Navier-Stokes solver [1, 2, 7, 8]. This simulation is done to model the experimental wakes shown in Figures $4 \mathrm{~b}, 5,6 \mathrm{~b}$, and $7 \mathrm{~b}$, where $\Gamma_{c} / \Gamma_{t}=-0.37$. 


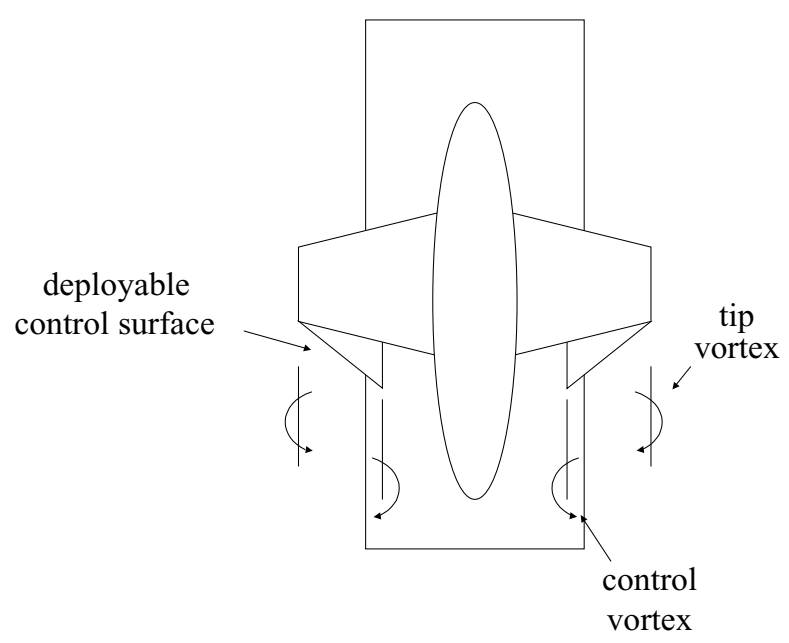

Figure 9: Implementation of PWVC to the diving planes of a submarine by means of deployable control surfaces. These control surfaces generate unequal strength, counter-rotating control vortices next to each of the tip vortices.

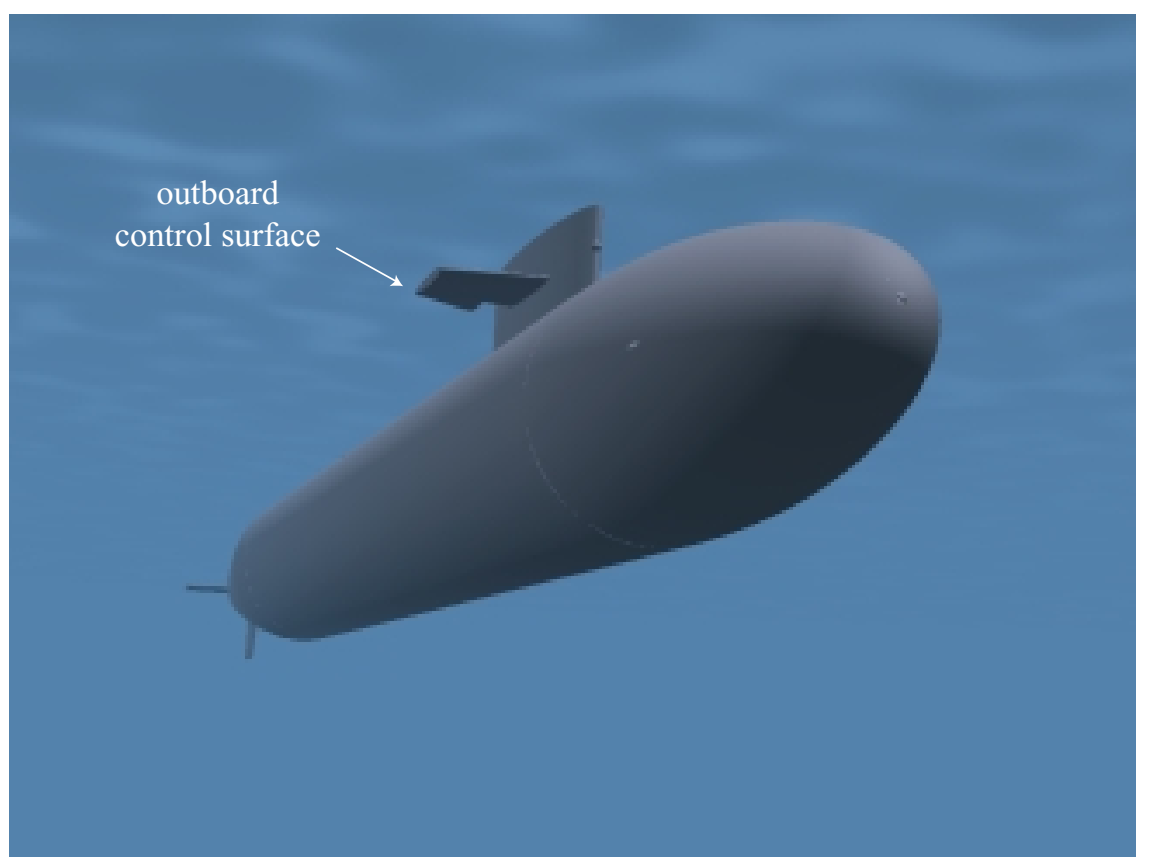

Figure 10: Conceptual rendering of a submarine using PWVC in a littoral environment. 


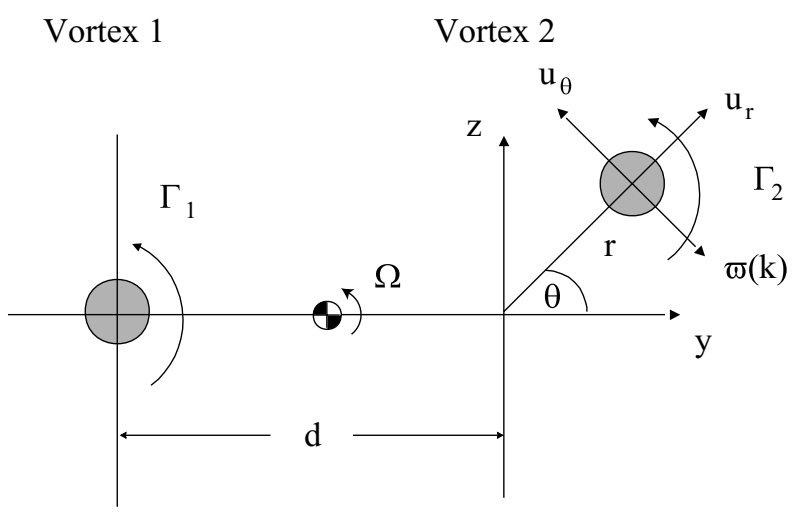

Figure 11: Cross-section of a single control (vortex 2) and tip (vortex 1) vortex pair. The sinusoidal perturbation amplitude on vortex 2 is given by $r$, where $r / d<<1$. The self-induced rotation rate of vortex 2 is given by $\varpi(k)$ and the radial and azimuthal components of the rate of strain field by $u_{r}$ and $u_{\theta}$, respectively. The vortices orbit about their common vorticity centroid at an angular velocity of $\Omega=\left(\Gamma_{1}+\Gamma_{2}\right) /\left(2 \pi d^{2}\right)$.

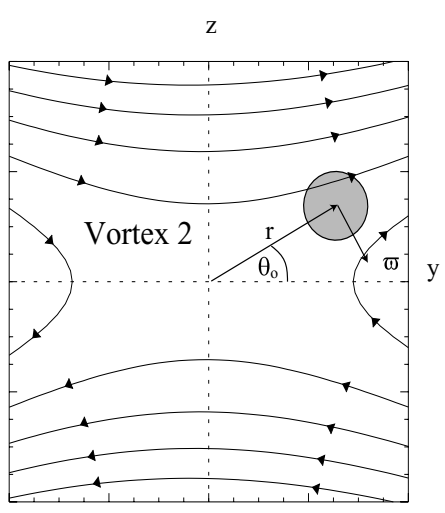

(a)

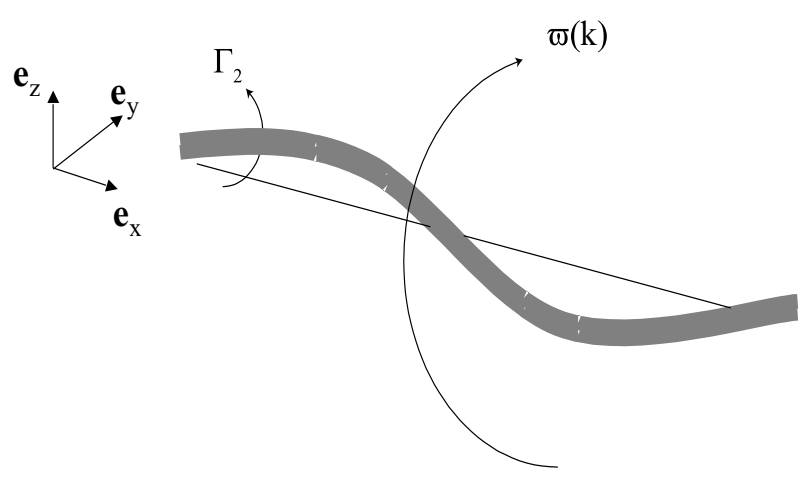

(b)

Figure 12: (a) Rate of strain field about vortex 2 for a counter-rotating vortex pair $\left(\Gamma_{1}<0\right.$ and $\Gamma_{2}>0$, $\left.\left|\Gamma_{1}\right| \neq\left|\Gamma_{2}\right|\right)$. (b) Self-induced rotation rate of vortex 2 is given by $\varpi(k)$ and rotates in a direction opposite (retrograde) to that of the flow about the vortex. 


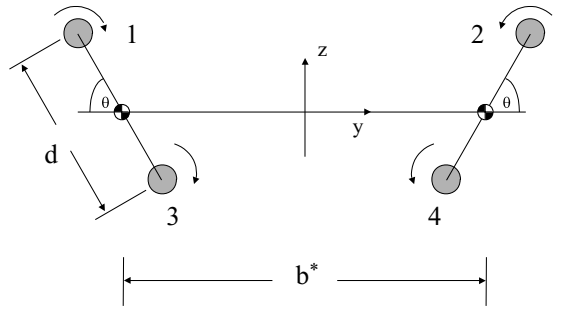

(a)

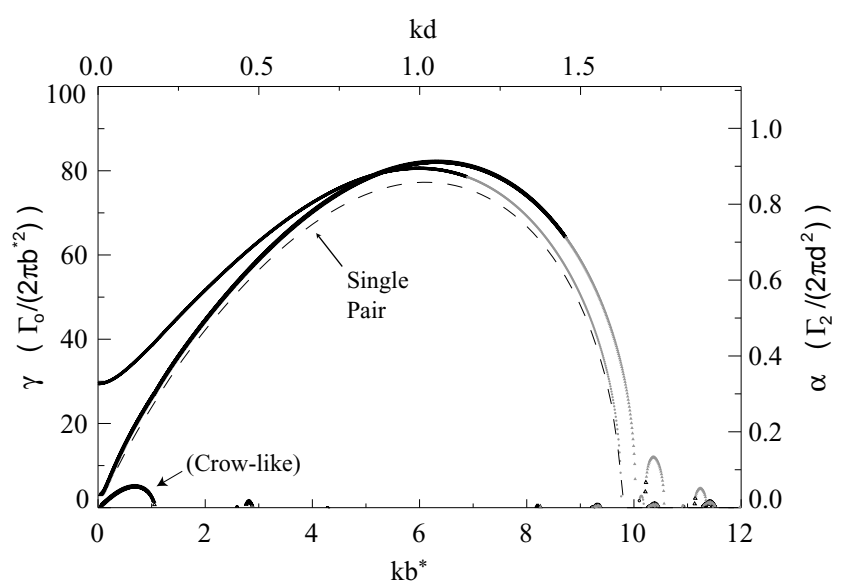

(b)

Figure 13: (a) Schematic of the vortex system used to analyze the stability properties of two counter-rotating vortex pairs. The distance between the vorticity centroids on either side of the wake is $b^{*}$ and the distance between the vortices within each pair by $d$. (b) Growth rate curves for two counter-rotating vortex pairs $\left(\Gamma_{4} / \Gamma_{2}=-0.6, d / b^{*}=0.1666\right)$ and for a single counter-rotating vortex pair (vortices 2 and $4, \Gamma_{4} / \Gamma_{2}=-0.6$ ). The dashed line indicates the growth rate curve for the single vortex pair, while the symbols indicate the growth rate curves for the four-vortex system. The perturbation wavenumber and growth rate are $k b^{*}$ and $\gamma$ for the four-vortex system and $k d$ and $\alpha$ for the single vortex pair, where $\Gamma_{o}=\Gamma_{2}+\Gamma_{4}$. 